\title{
INTERVALO HÍDRICO ÓTIMO NO PERFIL EXPLORADO PELAS RAÍZES DE FEIJOEIRO EM UM LATOSSOLO SOB DIFERENTES NÍVEIS DE COMPACTAÇÃO ${ }^{(1)}$
}

\author{
Douglas Rodrigo Kaiser ${ }^{(2)}$, Dalvan José Reinert ${ }^{(3)}$, José Miguel Reichert ${ }^{(3)}$, \\ Gilberto Loguércio Collares ${ }^{(4)} \&$ Marcelo Kunz ${ }^{(5)}$
}

\begin{abstract}
RESUMO
O intervalo hídrico ótimo (IHO) é integrador dos fatores de crescimento das plantas, e a densidade crítica obtida é um indicativo da qualidade estrutural do solo. O objetivo deste trabalho foi determinar o IHO em um Latossolo argiloso. Amostras de solo com estrutura preservada foram coletadas num experimento com três niveis de compactação: $\mathrm{PD}$ - plantio direto continuado por seis anos, $\mathrm{PDc}$ - plantio direto com compactação adicional e Esc-escarificação. Para a curva de resistência, coletaram-se 107 amostras na camada de 0 a $0,20 \mathrm{~m}$ em diferentes condições de umidade. Para a curva de retenção de água, coletaram-se amostras nas camadas de 0 a 0,$05 ; 0,05$ a 0,$10 ; 0,10$ a 0,$15 ; 0,20$ a 0,$25 ;$ e 0,30 a $0,35 \mathrm{~m}$. O modelo de resistência à penetração ajustado, com base na densidade e umidade, explicou $33 \%$ da variação obtida na resistência do solo à penetração, sendo todos os parâmetros de ajuste significativos. A densidade crítica do IHO é dependente do valor de resistência à penetração considerado limitante, sendo de 1,$36 ; 1,40 ; 1,45 ; \mathrm{e}$ $1,49 \mathrm{Mg} \mathrm{m}^{-3}$ para valores de $\mathrm{RP}$ de 1,$5 ; 2,0 ; 2,5 ; \mathrm{e} 3,0 \mathrm{MPa}$, respectivamente. A aeração do solo passa a ser limitante com densidades acima de $1,32 \mathrm{Mg} \mathrm{m}^{-3}$. A compactação do solo reduziu o seu IHO na camada próxima a $0,10 \mathrm{~m}$ de profundidade. Quando se adotou a resistência crítica de $2 \mathrm{MPa}$, o IHO foi nulo nas camadas de 0,05 a 0,12 m no PD, de 0,05 a 0,17 m no Esc e de 0,03 a 0,22 m no PDc. Com a utilização de 3 MPa como resistência crítica, ocorreu ampliação, em que o IHO tem valor positivo,
\end{abstract}

\footnotetext{
(1) Pesquisa executada com recursos do CNPq e FAPERGS. Recebido para publicação em outubro de 2008 e aprovado em maio de 2009.

${ }^{(2)}$ Doutorando em Ciência do Solo, Universidade Federal de Santa Maria- UFSM. Campus Universitário, CEP 97106-900 Santa Maria (RS). Bolsista CAPES. E-mail: kaiser@mail.ufsm.br

(3) Professor Titular do Departamento de Solos, Centro de Ciências Rurais, UFSM. E-mails: dalvan@ccr.ufsm.br; reichert@smail.ufsm.br

(4) Professor do Departamento de Ciências Agrárias, Conjunto Agrotécnico Visconde da Graça, Universidade Federal de Pelotas - CAVG/UFPEL. Av. Ildefonso Simões Lopes 2791, Bairro Arco Iris, CEP 96060-290 Pelotas (RS). E-mail: collares@ufpel.tche.br

${ }^{(5)}$ Mestrando em Engenharia Agrícola.UFSM. Bolsista CAPES. E-mail: mkunzcelo@hotmail.com
} 
no perfil do solo; o IHO foi nulo apenas na camada de 0,05 a 0,15 m do PDc. As raízes do feijoeiro não cresceram na camada de solo onde o IHO foi nulo com resistência crítica de 3 MPa.

Termos de indexação: plantio direto, densidade crítica, resistência à penetração, aeração.

\title{
SUMMARY: LEAST LIMITING WATER RANGE IN AN OXISOL PROFILE PENETRATED BY COMMON BEAN ROOTS UNDER DIFFERENT COMPACTION LEVELS
}

\begin{abstract}
The least limiting water range (LLWR) integrates the plant growth factors and the critical bulk density is an indicator of the soil structural quality. The objective of this study was to determine the LLWR of a clayey Oxisol. Soil samples with preserved structure were collected in an experiment with three levels of soil compaction: NT-continuous no-tillage for six years, $N T c-n o-t i l l a g e$ with additional compaction and Chi-chisel plowing on previous no-till soil. To determine soil penetration resistance 107 samples were collected in the 0-0.20 m layer, at different soil moisture levels, whereas for the soil water retention curve samples were collected in the layers $0-0.05,0.05-0.10,0.10-0.15,0.20-0.25$ and $0.30-0.35 \mathrm{~m}$. The adjusted soil penetration resistance model, based on bulk density and soil moisture, explained $33 \%$ of the variation observed in soil penetration resistance and all parameters were significant in this adjustment. The critical bulk density of the LLWR depends on the value of limiting soil penetration resistance, which was $1.36,1.40,1.45$, and $1.49 \mathrm{Mg} \mathrm{m}^{-3}$ for the RP values of 1.5 , 2.0, 2.5, and 3.0 MPa, respectively. Soil aeration became limiting when densities exceeded $1.32 \mathrm{Mg} \mathrm{m}^{-3}$. Soil compaction reduced LLWR in the layers around $0.10 \mathrm{~m}$. At a critical resistance of $2 \mathrm{MPa}, L L W R$ was zero in the layer 0.05-0.12 $\mathrm{m}$ for NT, in 0.05-0.17 $\mathrm{m}$ for Chi, and between 0.03 and $0.22 \mathrm{~m}$ for NTc. The use of $3 \mathrm{MPa}$ as critical resistance resulted in positive LLWR value in deeper soil layers, and was zero only in the 0.05-0.15 m layer of the NTc. Common bean roots did not grow in the soil layer where the LLWR was zero with a critical resistance of $3 \mathrm{MPa}$.
\end{abstract}

Index terms: no-tillage, critical density, penetration resistance, aeration.

\section{INTRODUÇÃO}

As propriedades físicas do solo são variáveis no tempo e no espaço, sendo alteradas pelas condições de manejo do solo e pelas variações climáticas ocorridas no período. O revolvimento ou a compactação do solo são as principais formas de alterar a sua estrutura, refletindo-se em modificações na sua densidade (Silva et al., 2004; Streck et al., 2004; Veiga et al., 2007) e na quantidade, continuidade e tamanho dos poros do solo (Klein \& Libardi, 2002). Devido a essas alterações, o solo apresentará comportamento diferenciado na resistência ao crescimento das raízes, nas trocas gasosas e na retenção e disponibilidade de água.

Para expressarem seu potencial produtivo, as plantas necessitam de suprimento adequado de água e oxigênio durante o seu ciclo de desenvolvimento. Para uma determinada condição estrutural, a umidade do solo é o principal fator que estabelecerá o momento em que a resistência do solo ou as trocas gasosas se tornam limitantes às plantas, sendo isso dependente das condições climáticas ocorridas no período. Em razão disso, uma determinada condição pode se tornar limitante ao crescimento das plantas, dependendo do conteúdo de água no solo e do período fenológico em que a planta se encontra. Em condições de alta umidade do solo, a aeração pode ser deficiente e prejudicar as trocas gasosas e a respiração das raízes das plantas (Drew, 1983; Morard \& Silvestre, 1996). Em condições de solo seco, o crescimento radicular pode ser restringido a determinadas camadas (Montagu et al., 2001) ou em fissuras ou bioporos do solo, devido à alta resistência oferecida pelas camadas compactadas. Por outro lado, a bioporosidade pode ser aumentada com o uso de plantas de cobertura (Abreu et al., 2004) com sistema radicular capaz de romper camadas compactadas e formar poros novos, contínuos e estáveis.

Vários atributos podem ser utilizados para caracterizar e qualificar a estrutura do solo, mas a resposta das plantas dependerá da interação entre eles (Reichert et al., 2003). Dessa forma, torna-se difícil estabelecer de forma isolada o efeito desses atributos sobre o crescimento das plantas. Com o objetivo de 
integrar as principais propriedades físicas do solo determinantes do crescimento das plantas, Silva et al. (1994) propuseram o intervalo hídrico ótimo (IHO) do solo, baseado nos pressupostos de Letey (1985), como um índice para avaliar a qualidade física do solo.

Esse índice pode ser considerado um "semáforo" da qualidade estrutural do solo. As condições de umidade ideais, para o desenvolvimento e crescimento das plantas, ocorrem entre os limites superior e inferior do IHO. As condições são limitantes quando ficam acima ou abaixo dos limites do IHO, e críticas ao crescimento das plantas quando a densidade do solo estiver acima da densidade onde o IHO é nulo (Silva \& Kay, 1996, 1997; Collares et al., 2006). A densidade crítica (IHO é nulo) obtida por meio do IHO auxilia na tomada de decisões diante das condições de manejo adotadas ou a serem adotadas em determinado solo.

A densidade crítica é variável em função do nível de compactação e da textura do solo (Wu et al. 2003; Reichert et al., 2007, 2009); solos com alta densidade atingem altos níveis de resistência à penetração com menor variação de umidade, reduzindo o crescimento radicular das plantas (Goodman \& Ennos, 1999). Além disso, as plantas, quando encontram condições adequadas de crescimento no início do seu ciclo vegetativo, tendem a manter o sistema radicular superficialmente. No entanto, a partir do momento que ocorrem períodos de estiagem, o acesso à água é limitado, pois o solo seco e compactado oferece alta resistência à penetração das raízes (Clark et al., 2003), não permitindo o aproveitamento adequado da água armazenada nas camadas mais profundas do solo ou abaixo da camada de maior resistência. Quando a umidade do solo fica fora dos limites do IHO durante o seu ciclo vegetativo, ocorre menor crescimento radicular e da parte aérea (Silva \& Kay, 1996; Collares et al., 2006), com possível redução na produtividade das culturas.

Por sua vez, solos bem estruturados, com densidade abaixo da crítica e com macroporosidade acima de $10 \%$, apresentam menor efeito sobre o crescimento radicular, mesmo que a resistência do solo à penetração esteja acima de $2 \mathrm{MPa}$ (Collares et al., 2006), pois os poros representam os caminhos preferenciais ao crescimento das raízes (Stirzaker et al., 1996; Rasse \& Smucker, 1998), permitindo que as camadas mais profundas do solo sejam ocupadas na busca de água e nutrientes. Dessa forma, nem sempre se observa resposta das plantas quando o solo mantém resistência de $2 \mathrm{MPa}$ por determinado período (Silva, 2003).

O efeito da alta resistência vai depender também da fase em que a cultura se encontra. O crescimento radicular normalmente cessa a partir do florescimento das plantas e, nesse caso, a deficiência de água e de oxigênio poderá ser o principal fator limitante à produção das culturas, visto que as plantas já possuem o seu desenvolvimento alterado quando a água atinge potenciais menores que -1,5 MPa (Taiz \& Zieger, 2004).
Este estudo teve como objetivo determinar o intervalo hídrico ótimo, considerando diferentes valores de resistência crítica, para um Latossolo Vermelho sob diferentes níveis de compactação.

\section{MATERIAL E MÉTODOS}

Para a realização deste estudo, amostras de solo com estrutura preservada foram coletadas em experimento instalado na área do Campo Experimental da Agronomia da UNICRUZ, no município de Cruz Alta, no Rio Grande do Sul. O solo foi um Latossolo Vermelho (Embrapa, 1999) com $607 \mathrm{~g} \mathrm{~kg}^{-1}$ de argila, $176 \mathrm{~g} \mathrm{~kg}^{-1}$ de silte e $217 \mathrm{~g} \mathrm{~kg}^{-1}$ de areia na profundidade de $0,5 \mathrm{~m}$.

A coleta das amostras foi feita nas parcelas de um experimento conduzido sob o delineamento experimental de blocos ao acaso com três repetições, em que os tratamentos foram diferenciados em níveis de compactação: PD - plantio direto continuado, que reflete o histórico das pressões aplicadas por seis anos de manejo sob plantio direto; PDc - plantio direto com compactação adicional, que visou aumentar o estado de compactação da área; e Esc - escarificação efetuada após seis anos sob PD, para reduzir a compactação atual do solo. A compactação adicional das parcelas (PDc) foi realizada no dia 14 de janeiro de 2003, por meio de quatro passadas paralelas e sobrepostas de uma pá carregadeira articulada Caterpilar 966, com rodado de pneus, com massa total de $16,6 \mathrm{Mg}$. A escarificação foi realizada com um escarificador de sete hastes até a profundidade de $0,25 \mathrm{~m}$; posteriormente, efetuou-se o nivelamento superficial do terreno, com uma grade de 36 discos. No experimento, foi implantada a cultura do feijoeiro em janeiro de 2003 e, em sucessão, a cultura do trigo. As amostras de solo foram coletadas no dia 24 de fevereiro de 2003 (40 dias após a semeadura do feijoeiro) e, no florescimento do feijoeiro, avaliou-se a distribuição do sistema radicular, usando o método do perfil (Collares et al., 2008)

Para determinar a curva de resistência do solo à penetração, 107 amostras de solo com estrutura preservada foram coletadas com anéis de Uhland de 0,075 $\mathrm{m}$ de diâmetro e altura, nas camadas de 0 a 0,10 e 0,10 a $0,20 \mathrm{~m}$, nos diferentes tratamentos, obtendo-se uma variação da densidade das amostras. Para obter variação de umidade, as amostras foram coletadas em diferentes épocas, conforme a ocorrência de ciclos de umedecimento e secagem do solo. No laboratório, essas amostras foram preparadas e submetidas à avaliação quanto à resistência à penetração, utilizando-se um penetrômetro com taxa constante de penetração e com área basal de $129 \mathrm{~mm}^{2}$, diâmetro da base de $12 \mathrm{~mm}$ e ângulo de $30^{\circ}$ (Reinert et al., 2007).

Os dados de resistência do solo à penetração foram ajustados em função da densidade do solo e da umidade 
volumétrica, utilizando-se o modelo não linear proposto por Busscher (1990) e seguindo-se os procedimentos descritos em Silva et al. (1994). O coeficiente de determinação $\left(\mathrm{R}^{2}\right)$ do modelo não linear foi calculado pela equação 1 .

$$
R^{2}=1-\left(\frac{S Q_{\text {RESIDUO }}}{S Q_{\text {REGRESSÃO }}}\right)
$$

Para definir o intervalo hídrico ótimo, utilizaramse os valores de 1,5; 2,0; 2,5; e 3,0 MPa como limitantes da resistência à penetração, obtendo-se, dessa forma, uma densidade crítica para cada curva de resistência obtida. Em condições estruturais naturais, o solo apresenta como limite superior a umidade na capacidade de campo $\left(\theta_{\mathrm{CC}}\right)$ e, como limite inferior, a umidade no ponto de murcha permanente $\left(\theta_{\mathrm{PMP}}\right)$ (Letey, 1985). A densidade crítica corresponde ao valor de densidade no qual o IHO é nulo, sendo definida pela interseção das equações que determinam os limites superiores e inferiores do IHO. A definição desses limites permite obter diferentes valores de densidade crítica, que podem se tornar referência conforme a suscetibilidade das diferentes espécies agrícolas às restrições ao crescimento radicular. Normalmente, o valor de $2 \mathrm{MPa}$ é definido como crítico; no entanto, muitas vezes não se observa resposta das culturas agrícolas quando o solo atinge esses valores (Collares et al., 2006; Reichert et al., 2007).

Para determinar a curva de retenção de água e definir a umidade na capacidade de campo e no ponto de murcha permanente, as amostras de solo com estrutura preservada foram coletadas nas camadas de 0 a 0,$05 ; 0,05$ a 0,$10 ; 0,10$ a 0,$15 ; 0,20$ a 0,25 ; e 0,30 a $0,35 \mathrm{~m}$, com anéis de $0,054 \mathrm{~m}$ de diâmetro e $0,03 \mathrm{~m}$ de altura. Foram utilizadas três técnicas para obtenção dos pontos da curva de retenção de água: a mesa de tensão, para os potenciais de -0,001, $-0,006 \mathrm{e}$ -0,01 MPa (Reinert \& Reichert, 2006); a câmara de pressão de Richards (panelas de pressão), para os potenciais de -0,033 e -0,1 MPa (Klute, 1986); e o WP4 (Dewpoint PotentiaMeter), para os potenciais de -0,5 e - $1,5 \mathrm{MPa}$ (Klein et al., 2006). A partir da curva de retenção de água, obteve-se a umidade do solo na capacidade de campo (-0,01 MPa) e no ponto de murcha permanente (-1,5 MPa). No entanto, como a umidade no potencial de $-1,5 \mathrm{MPa}$ foi obtida a partir de amostras deformadas e em base gravimétrica, multiplicou-se o seu valor pela densidade do solo para se obter a umidade volumétrica. A umidade do solo na capacidade de campo e no ponto de murcha permanente foi ajustada em função da densidade do solo por meio de regressões, utilizando-se o pacote estatístico SAS (SAS, 1991).

\section{RESULTADOS E DISCUSSÃO}

Os atributos físicos do solo, avaliados para determinação do intervalo hídrico ótimo (IHO), indicam que a maior variação (CV) ocorreu na resistência à penetração, devido à variação da densidade e da umidade do solo (Quadro 1). O coeficiente de variação encontrado é menor que os observados em trabalhos onde o gradiente de umidade é condicionado em laboratório (Tormena et al., 1998). Parte disso deve-se também à área de contato do cone do penetrômetro utilizado, pois penetrômetros com maior área de contato, mesmo mantendo o mesmo ângulo, possuem menor influência dos poros do solo na resistência mecânica à penetração (Lowery \& Morrison, 2002).

$\mathrm{O}$ modelo de resistência à penetração ajustado (Equação 2), na sua forma linearizada, explicou $33 \%$ da variação obtida na resistência do solo à penetração, sendo todos os parâmetros de ajuste altamente significativos (Quadro 2). No entanto, ao se utilizar a equação na sua forma não linear, observou-se que o coeficiente de determinação estimado pela equação 1 , a partir da análise da variância indicada para modelos não lineares (Schneider et al., 2009), passou a explicar $94 \%$ da variação da resistência à penetração (Quadro 3). Dessa forma, é importante indicar a forma de estimar a precisão dos modelos obtidos na determinação do IHO, pois modelos não lineares normalmente apresentam maior coeficiente de determinação (Tormena et al., 1998).

$$
\mathrm{RP}=0,07912 \mathrm{Ds}^{4,0123} \times \theta^{-1,9328}
$$

Quadro 1. Estimadores dos parâmetros para as variáveis físicas do solo determinadas nas amostras com estrutura preservada

\begin{tabular}{cccccc}
\hline Variável $^{(1)}$ & Média & Desvio-padrão & Mínimo & Máximo & CV \\
\hline RP & 1,758 & 0,496 & 0,814 & 3,080 & 28,15 \\
Ds & 1,290 & 0,051 & 1,140 & 1,410 & 3,930 \\
$\theta$ & 0,350 & 0,028 & 0,211 & 0,412 & 7,864
\end{tabular}

(1) RP: resistência do solo à penetração $(\mathrm{MPa})$; $\theta$ : umidade volumétrica do solo $\left(\mathrm{m}^{3} \mathrm{~m}^{-3}\right)$; Ds: densidade do solo $\left(\mathrm{Mg} \mathrm{m}^{-3}\right)$; $\mathrm{CV}$ : coeficiente de variação (\%); n: 107. 
A umidade do solo a partir da qual a resistência à penetração atingiu valores de 1,$5 ; 2,0 ; 2,5 ;$ e $3,0 \mathrm{MPa}$ foi calculada pela equação 3 , a qual representa um rearranjo da equação 2 .

$$
\theta=\left(\frac{R P}{0,07912 D s^{4,0123}}\right)^{\left(\frac{1}{1,9328}\right)}
$$

Os coeficientes dos modelos de retenção de água encontram-se nos quadros 4 e 5 . Para a umidade na capacidade de campo (-0,01 MPa), o modelo linear gerado explicou $15 \%$ da variabilidade (Quadro 4), enquanto o modelo linear obtido para estimar a umidade no ponto de murcha permanente $(-1,5 \mathrm{MPa})$ explicou $50 \%$ da variabilidade (Quadro 5).

Quadro 2. Estimadores dos parâmetros da regressão linearizada, para a resistência à penetração $(\mathrm{RP})$, em função da umidade volumétrica do solo $(\theta)$ e da densidade do solo (Ds): $\log _{10} \mathrm{RP}=\log _{10} a+b$ $\log _{10} \theta+c \log _{10} D s$

\begin{tabular}{cccccc}
\hline Parâmetro & Erro-padrão & $\mathbf{t}$ & Probabilidade $^{(1)}$ & $\mathbf{R}^{2}$ \\
\hline$a$ & $-1,1017$ & 0,1941 & $-5,67$ & 0,0001 & 0,33 \\
$b$ & $-1,9328$ & 0,3197 & $-6,04$ & 0,0001 & \\
$c$ & 4,0123 & 0,6817 & 5,89 & 0,0001 &
\end{tabular}

(1) Probabilidade de significância para os valores estimados dos parâmetros.

Quadro 3. Quadro da análise da variância do modelo não linear

$\begin{array}{llllllll}\text { Fonte de variação } & \text { GL } & \text { SQ } & \text { QM } & \text { F } & \text { Pr }>\text { F } & \mathbf{R}^{2}\end{array}$

$\begin{array}{lrrrrr}\text { Regressão } & 3 & 324,8 & 108,3 & 580,9<0,0001 & 0,94 \\ \text { Resíduo } & 100 & 18,6 & 0,18 & \\ \text { Total não corrigido } & 103 & 343,4 & - & \\ \text { Total corrigido } & 102 & 24,9 & - & \end{array}$

GL: graus de liberdade; SQ: somatório ao quadrado; QM: quadrado médio.

Quadro 4. Estimadores dos parâmetros da regressão linear, para a umidade na capacidade de campo ( $\theta$ ) no potencial de $-0,01 \mathrm{Mpa}$, em função da densidade do solo (Ds): $\theta \mathrm{cc}=0,2494+0,1222 \mathrm{Ds}$

\begin{tabular}{cccccc}
\hline Parâmetro & Erro-padrão & $\mathbf{t}$ & Probabilidade $^{(1)}$ & $\mathbf{R}^{2}$ \\
\hline$a$ & 0,2494 & 0,06323 & 3,94 & 0,0003 & 0,15 \\
$b$ & 0,1222 & 0,04620 & 2,65 & 0,0116 & \\
\hline
\end{tabular}

(1) Significância para os valores estimados dos parâmetros. Sxy $=0,03 ; \mathrm{CV}=7,19 \%$.
Quadro 5. Estimadores dos parâmetros da regressão linear, para a umidade no ponto de murcha permanente $(\theta)$ no potencial de $-1,5 \mathrm{Mpa}$, em função da densidade do solo (Ds): $q \theta_{\mathrm{PMP}}=$ $0,04513+0,19819$ Ds

\begin{tabular}{cccccc}
\hline \multicolumn{1}{c}{ Parâmetro } & Erro-padrão & $\mathbf{t}$ & Probabilidade $^{(1)}$ & $\mathbf{R}^{2}$ \\
\hline$a$ & $-0,04513$ & 0,04118 & $-1,10$ & 0,2792 & 0,50 \\
$b$ & 0,19819 & 0,03002 & 6,60 & 0,0001 &
\end{tabular}

(1) Significância para os valores estimados dos parâmetros. Sxy $=0,02 ; \mathrm{CV}=8,75 \%$.

O IHO para o Latossolo em estudo, representado pela área hachurada, indica a amplitude de umidade do solo em que são mínimas as limitações ao desenvolvimento das plantas (Figura 1). Os limites superiores do IHO foram a umidade na capacidade de campo $\left(\theta_{\mathrm{CC}}\right)$ e o espaço aéreo do solo $\left(\theta_{10} \%\right)$. A porosidade de aeração passou a ser limitante com densidades acima de $1,32 \mathrm{Mg} \mathrm{m}^{-3}$ (Figura 1). Os limites inferiores do IHO foram a umidade no ponto de murcha permanente $\left(\theta_{\mathrm{PMP}}\right)$ e a resistência do solo à penetração $\left(\theta_{\mathrm{RP}}\right)$. A $\theta_{\mathrm{PMP}}$ só passa a ser limitante para densidades abaixo de 1,05, 1,17 e 1,28 $\mathrm{Mg} \mathrm{m}^{-3}$, quando o limite de $\mathrm{RP}$ adotado é 2,0, 2,5 e 3,0 MPa, respectivamente, sendo esses valores de densidade baixos em relação aos de Latossolos utilizados para produção agrícola em plantio direto (Secco et al., 2004) e pouco frequentes em Latossolos sob floresta nativa (Fontanela, 2008). A densidade em que o IHO passou a ser nulo variou com o valor de resistência considerado restritivo. Os valores de densidade crítica foram de 1,36; 1,40; 1,45; e $1,49 \mathrm{Mg} \mathrm{m}^{-3}$, quando os níveis críticos de resistência à penetração adotados foram de 1,$5 ; 2,0 ; 2,5$; e 3,0 $\mathrm{MPa}$, respectivamente.

Para cada condição de densidade do solo, a resistência do solo à penetração passará a ser controlada pelos ciclos de umedecimento e secagem do solo. Para cada valor de densidade crítica estabelecida no IHO (Figura 1), a resistência do solo tem aumento acentuado à medida que a umidade do solo fica abaixo da capacidade de campo (Figura 2). Isso indica que, mesmo o solo apresentando densidades baixas, a resistência dele à penetração pode restringir o crescimento radicular das plantas quando a umidade do solo ficar próxima ao ponto de murcha permanente. Dessa forma, a duração dos ciclos de umedecimento e secagem do solo altera diretamente a resistência, a aeração e a temperatura do solo, que são fatores de crescimento das plantas (Letey, 1985); o efeito de cada fator dependerá da fase de desenvolvimento em que a planta se encontra. Além disso, à medida que o solo vai secando, a disponibilidade de água reduz e, nessas condições, a planta encontra maior dificuldade para o crescimento aéreo e radicular, pois a turgescência celular é reduzida nos meristemas de crescimento 

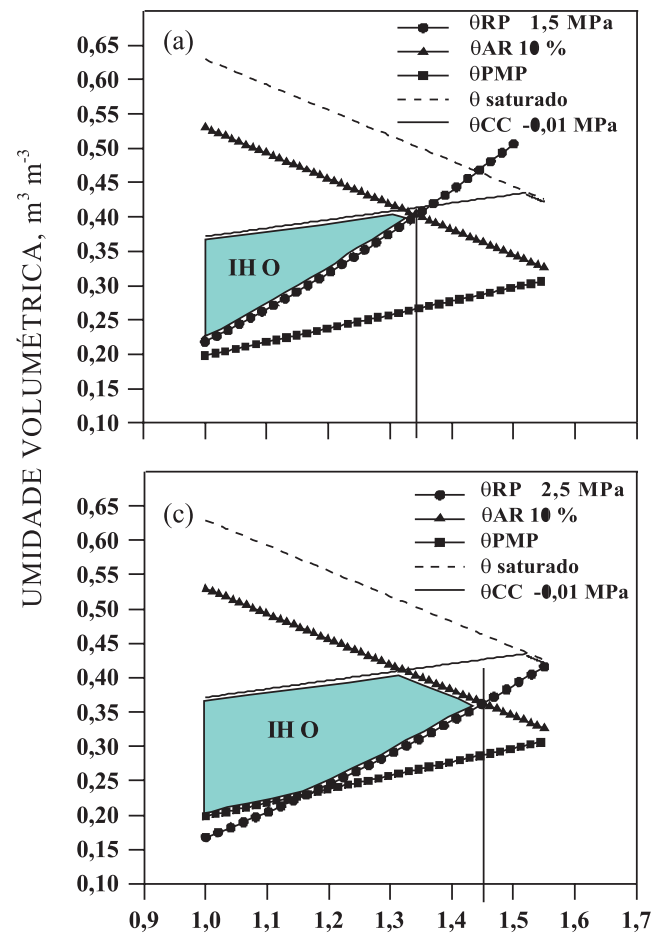
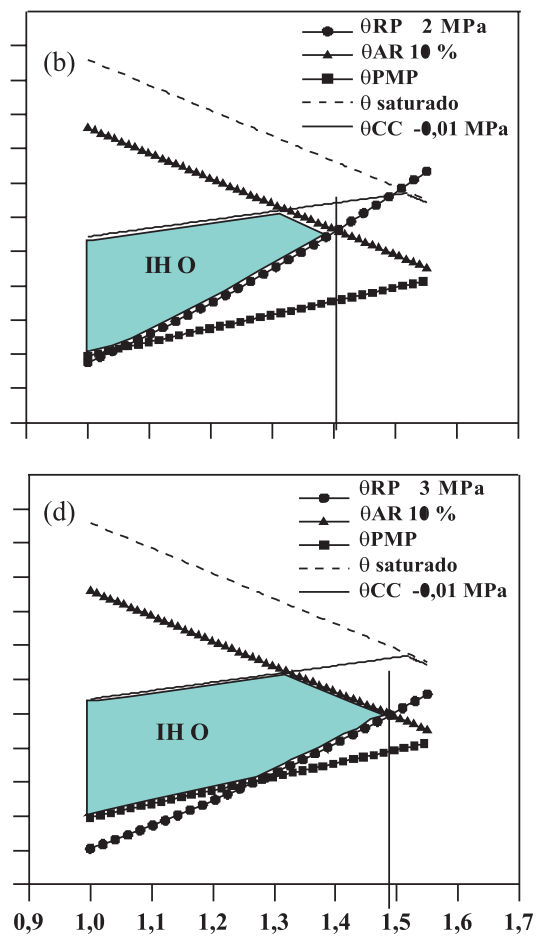

DENSIDADE DO SOLO, $\mathrm{Mg} \mathrm{m}^{-3}$

Figura 1. Variação do intervalo hídrico ótimo (IHO) em função da resistência do solo à penetração considerada crítica.

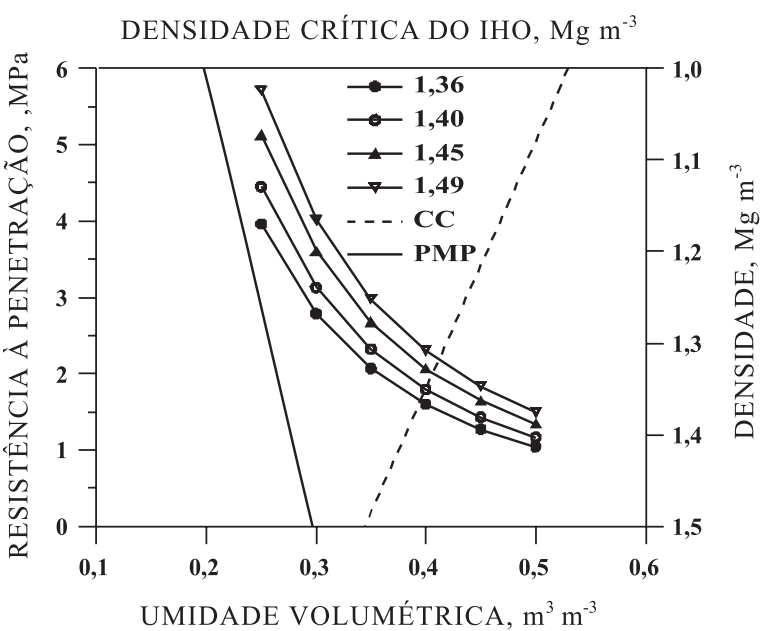

Figura 2. Variação da resistência do solo à penetração em função da umidade volumétrica, considerando a densidade crítica do solo obtida no intervalo hídrico ótimo. CC: umidade na capacidade de campo; PMP: umidade no ponto de murcha permanente.

(Davies \& Zhang, 1991). É fundamental para o crescimento da planta que o solo apresente condições favoráveis ao crescimento das raízes, o que permite explorar um maior volume de solo em profundidade, aumentando o acesso à água e reduzindo os riscos de deficiência hídrica.
A resistência do solo à penetração tem sido apontada como a principal restrição física ao crescimento radicular das plantas (Streck, 2003; Collares et al., 2006), porém o seu efeito sobre o sistema radicular só tem sido verificado quando os períodos de alta resistência do solo ocorrem antes do florescimento das plantas (Riquelme, 2004), que corresponde à fase de maior crescimento das raízes. As raízes das plantas exercem uma pressão de crescimento de 0,2 a 1,2 MPa (Bengough \& Mullins, 1990; Clark et al., 1999, 2003). A força motora do crescimento radicular é a pressão de turgescência celular na zona de elongação celular da raiz, sendo fundamental a disponibilidade de água para a ocorrência desse processo (Bengough et al., 2006). Portanto, a capacidade de uma planta vencer a resistência do solo depende da espessura da raiz e da sua habilidade em sintetizar substâncias mucilaginosas na coifa, que atuarão como lubrificantes, facilitando o crescimento (Bengough et al., 2006); dessa forma, o valor de $2 \mathrm{Mpa}$, corriqueiramente adotado como restritivo (Silva et al., 1994; Tormena et al., 1998; Collares et al., 2006), pode não representar restrições às plantas (Silva, 2003; Klein \& Camara, 2007), desde que haja disponibilidade de água às plantas. Durante o ciclo do feijoeiro, não ocorreu deficiência hídrica (Collares et al., 2008) e o crescimento radicular não foi restringido (Figura 7) com densidades abaixo de $1,4 \mathrm{Mg} \mathrm{m}^{-3}$, quando se adotou $2 \mathrm{MPa}$ como resistência crítica (Figura 5).

Os valores de densidade crítica de $1,4 \mathrm{Mg} \mathrm{m}^{-3}$ estão próximo daqueles determinados em Latossolos argilosos por outros autores. Nesse sentido, Leão et 
al. (2004) encontraram densidade crítica de $1,43 \mathrm{Mg} \mathrm{m}^{-3}$ num Latossolo com 40 \% de argila, na região de Campo Grande - MS, enquanto Klein \& Camara (2007) encontraram o valor de $1,34 \mathrm{Mg} \mathrm{m}^{-3}$ de densidade crítica para um Latossolo com $61 \%$ de argila, na região de Passo Fundo - RS, independentemente do manejo adotado. Esses autores observaram também que a $\mathrm{RP}$ foi o limite inferior, e a $\theta_{\mathrm{CC}}$ e a aeração, os limites superiores do IHO. As variações nos valores de densidade crítica ocorrem devido às diferenças no teor de argila entre os Latossolos, concordando com Reichert et al. (2007, 2009), que constataram uma relação direta entre o teor de argila e a densidade crítica obtida pelo IHO.

A variação do IHO com a densidade tem redução mais acentuada quando a $\mathrm{RP}$ crítica é de $1,5 \mathrm{MPa}$ (Figura 3). O valor máximo do IHO foi de $0,173 \mathrm{~m}^{3} \mathrm{~m}^{-3}$ quando a RP crítica foi assumida como $2 \mathrm{MPa}$. A

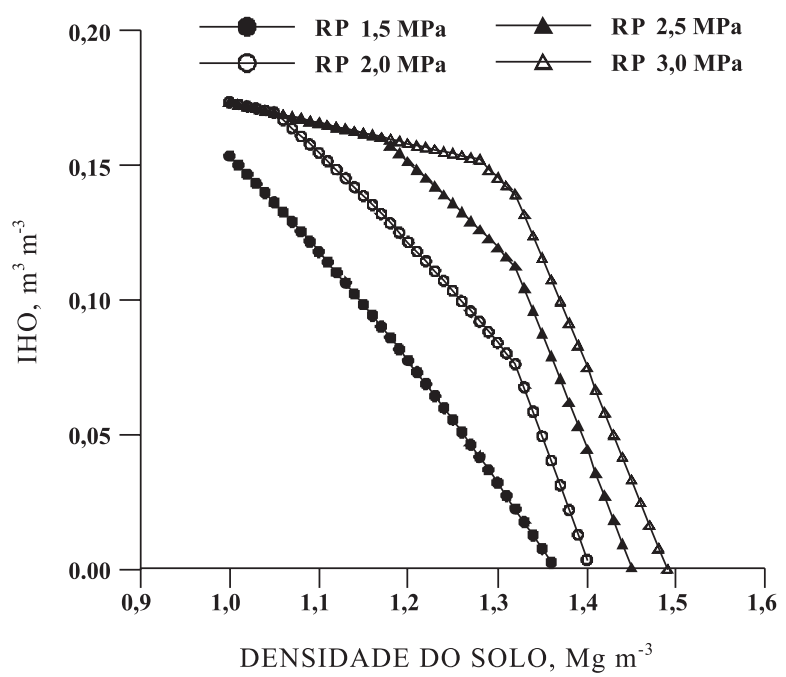

Figura 3. Variação do intervalo hídrico ótimo (IHO) em função da resistência do solo à penetração considerada crítica. umidade do solo onde a sua resistência é considerada restritiva é o fator que mais reduz a amplitude do IHO em condições de solos agrícolas (Imhoff et al., 2001), por apresentar maior variação com a alteração da densidade do solo. À medida que aumenta a densidade do solo, o IHO reduz, pois ocorre aumento acentuado da resistência à penetração com a menor variação da umidade do solo. Em Latossolo sob mata nativa, Fontanela (2008) observou que a $\theta_{\mathrm{PMP}}$ foi o limite inferior do IHO e, em áreas agrícolas, a RP. Dessa forma, o revolvimento e o tráfego de máquinas alteram a estrutura do solo, aumentando a densidade e reduzindo a macroporosidade do solo; nessas condições, as limitações físicas ao crescimento de plantas podem ser atingidas com menores variações no conteúdo de água do solo, e a resposta da planta vai depender da magnitude e da duração da resistência oferecida pelo solo (Bengough et al., 2006).

No Latossolo em estudo, para densidades acima de $1,32 \mathrm{Mg} \mathrm{m}^{-3}$, o espaço aéreo passa a ser o limite superior do IHO, indicando que, a partir desse valor, a porosidade do solo começa a ser alterada pela compactação, principalmente os macroporos, os quais são os principais condutores de ar no solo (Kaiser et al., 2008), podendo limitar as trocas gasosas e prejudicar as plantas pela deficiência de oxigênio ou excesso de $\mathrm{CO}_{2}$. Em períodos de maior umidade, os solos compactados, por possuírem menor quantidade e continuidade de poros, podem apresentar deficiência de oxigênio para as raízes das plantas (Lapen et al., 2004). Além da falta de oxigênio (Drew, 1983), o aumento na concentração de $\mathrm{CO}_{2}$ no ambiente radicular afeta as plantas (Boru et al., 2003; Greenway et al., 2006).

Apesar de o IHO indicar que, com densidades acima de $1,32 \mathrm{Mg} \mathrm{m}^{-3}$, a aeração passa a ser limitante em condições de campo, observa-se que em todos os níveis de compactação a quantidade de macroporos é semelhante no perfil (Figura 4), existindo uma redução na camada próxima aos $0,10 \mathrm{~m}$, onde ocorre aumento

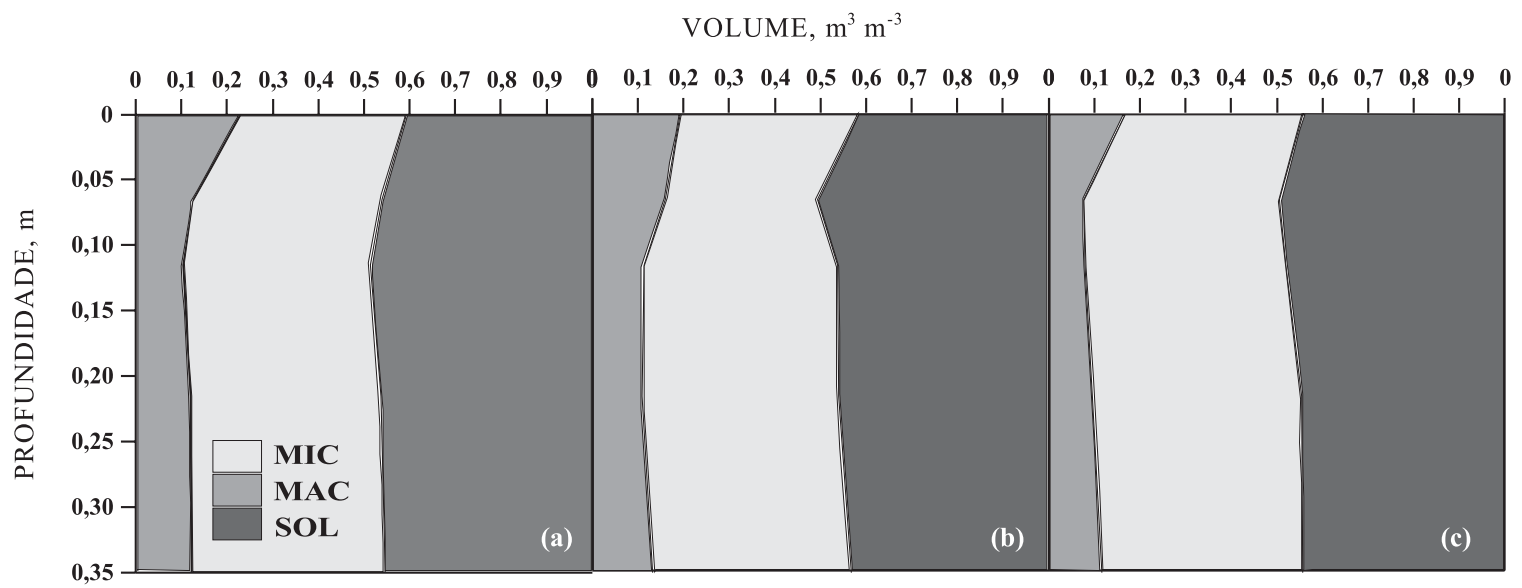

Figura 4. Caracterização da estrutura e da distribuição de poros no perfil do Latossolo nos três níveis de compactação: (a) plantio direto contínuo; (b) escarificação; e (c) plantio direto com compactação adicional. MAC: macroporos; MIC: microporos; SOL: fração sólida. 
do grau de compactação. Em solos sob plantio direto, a resistência à penetração tem sido o limite inferior do IHO e a aeração o limite superior (Betz et al., 1998), pois, nessas condições, observa-se aumento da densidade do solo e redução da macroporosidade.

Quando se transferiram o conceito e os limites do IHO para o perfil do solo no campo, observou-se que, quando a RP crítica adotada foi de $2 \mathrm{MPa}$, em todas as condições de manejo do solo ocorreu uma camada onde o IHO é nulo (Figura 5), diferenciando-se apenas o início e a espessura dessa camada e a variação do IHO. O PD apresentou a menor camada limitante e maior variação entre os limites do IHO, ao passo que, com a compactação do solo (PDc), observa-se antecipação e aumento na profundidade da camada limitante, o que se deve ao aumento da densidade do solo devido ao tráfego de máquinas (Collares et al.,

UMIDADE VOLUMÉTRICA, $\mathrm{m}^{3} \mathrm{~m}^{-3}$


Figura 5. Variação do intervalo hídrico ótimo (IHO) no perfil do solo, considerando $2 \mathrm{MPa}$ como a resistência limitante.
2008). Essa camada limitante, ocasionada pela compactação, também apareceu na área escarificada, o que pode ser atribuído à dificuldade de coleta de amostras com estrutura preservada em condições de solo mobilizado; isso normalmente induz a coleta nas zonas menos alteradas, as quais não sofreram desestruturação pela haste do escarificador devido à sua alta resistência ou compactação e, dessa forma, isso se reflete em maior densidade.

Ao se adotar o valor de $3 \mathrm{MPa}$ como resistência limitante, observou-se que, apenas com a compactação do solo (PDc), o IHO foi nulo na camada de 0,05 a $0,15 \mathrm{~m}$ de profundidade (Figura 6), indicando a degradação da estrutura pela ação das pressões dos pneus com o tráfego de máquinas agrícolas. No entanto, em todos os manejos, ocorreu a amplificação do IHO ao se mudar a RP crítica, semelhantemente

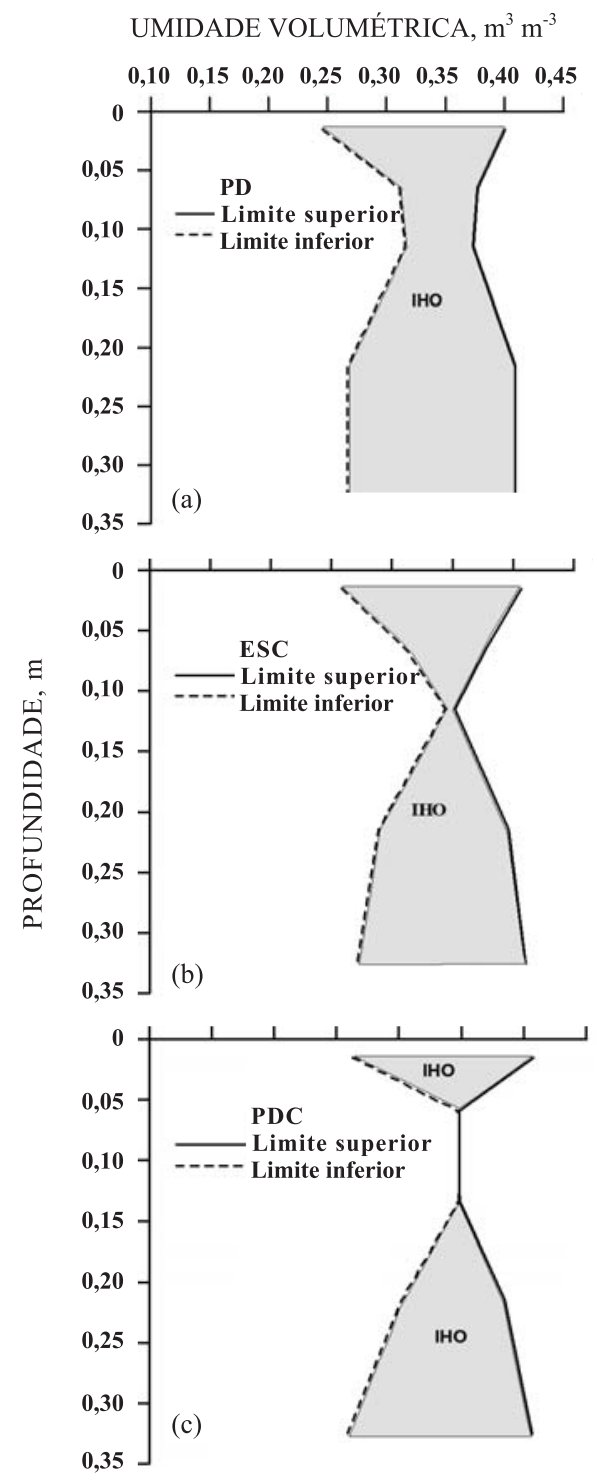

Figura 6. Variação do intervalo hídrico ótimo (IHO) no perfil do solo, considerando $3 \mathrm{MPa}$ como a resistência limitante. 
ao observado por Klein \& Camara (2007) em solo sob plantio direto e escarificação.

Mesmo que o solo apresentasse seus limites fora do IHO nas camadas de 0,05 a 0,15 m no PD e ESC, houve crescimento de raízes abaixo dessas camadas (Figura 7). Isso indica que o valor de $2 \mathrm{MPa}$ pode não restringir o crescimento radicular quando o solo apresenta macroporos contínuos, que são o caminho preferencial para o crescimento de raízes (Stirzaker et al., 1996; Rasse \& Smucker, 1998) e para o fluxo de água e gases no solo.

Apenas no PDc houve restrição do crescimento radicular até a camada em que o IHO é nulo (Collares et al., 2008). As camadas compactadas e com baixa porosidade definem o limite do crescimento radicular das plantas (Montagu et al., 2001). Em sistema de plantio direto, essas camadas foram denominadas por Reichert et al. (2009) como "pé-de-plantio direto", à semelhança do conceito de "pé-de-arado" em solos preparados convencionalmente. Solos bem estruturados,

PD
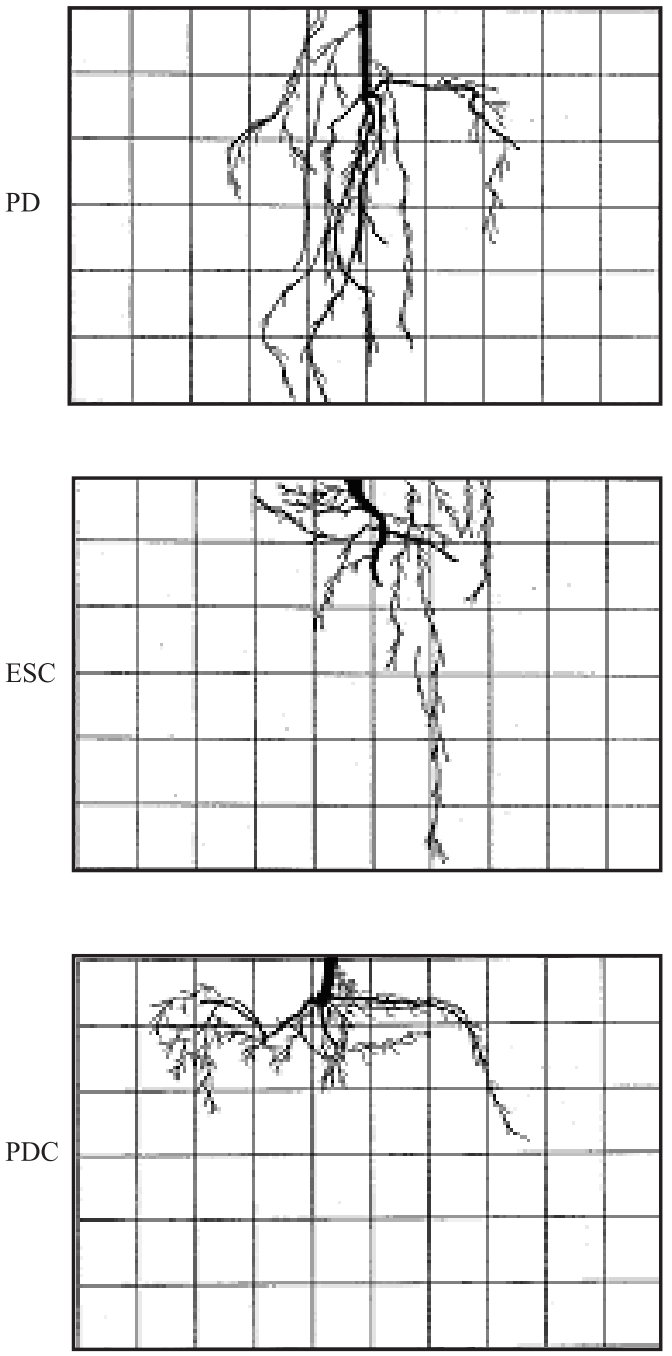

Figura 7. Distribuição radicular do feijoeiro sob diferentes níveis de compactação (Collares et al., 2008). por terem maior quantidade de poros grandes e contínuos, podem apresentar menor quantidade de água retida na capacidade de campo (-0,01 $\mathrm{MPa})$; nessas condições, o intervalo do IHO poderá ser reduzido nas amostras, mas poderá não representar restrições no campo.

\section{CONCLUSÕES}

1. A compactação do solo reduz o IHO no perfil do solo explorado pelas raízes, e a amplitude de variação e a densidade crítica obtida são dependentes do valor de resistência à penetração considerada limitante.

2. A resistência do solo à penetração e à aeração do solo são os principais fatores limitantes para esse Latossolo sob altos níveis de compactação.

3. As raízes do feijoeiro não cresceram na camada em que o IHO foi nulo com resistência crítica de $3 \mathrm{MPa}$.

\section{LITERATURA CITADA}

ABREU, S.L.; REICHERT, J.M. \& REINERT, D.J. Escarificação mecânica e biológica para a redução da compactação em Argissolo franco-arenoso sob plantio direto. R. Bras. Ci. Solo, 28:519-531, 2004.

BETZ, C.L.; ALlMARAS, R.R.; COPELAND, S.M. \& RANDALL., G.W. Least limiting water range traffic and long-term tillage influences in a Webster soil. Soil Sci. Soc. Am. J., 62:1384-1393, 1998.

BENGHOUGH, A.G.; BRANSBY, M.F.; HANS, J.; McKENNA, S.J.; ROBERTS, T.J. \& VALENTINE, T.A. Root responses to soil physical conditions: Growth dynamics from field to cell. J. Exper. Bot., 57:437-447, 2006.

BENGHOUGH, A.G. \& MULLINS, C.E. Mechanical impedance to root growth: A review of experimental techniques and root growth responses. J. Soil Sci., 41:341-358, 1990.

BORU, G.; VANTOAI, T.; ALVES, J.; HUA, D. \& KNEE, M. Responses of soybean to oxygen deficiency and elevated root-zone carbon dioxide concentration. Ann. Bot., 91:447453,2003

BUSSCHER, W.J. Adjustment of flat-tipped penetrometer resistance data to a common water content. Trans. Am. Soc. Agric. Eng., 33:519-524, 1990.

CLARK, L.J. \& BARRACLOUGH, P.B. Dicotyledons generate greater maximum axial root growth pressures than monocotyledons? J. Exp. Bot., 50:1263-1266, 1999.

CLARK, L.J.; WHALLEY, W.R. \& BARRACLOUGH, P.B. How do roots penetrate strong soil ? Plant Soil, 255:93-104, 2003.

COLLARES, G.L ; REINERT, D J * REICHERT, J M \& KAISER, D.R. Qualidade física do solo na produtividade da cultura do feijoeiro num Argissolo. Pesq. Agropec. Bras., 41:1663-1674, 2006.

COLLARES, G.L.; REINERT, D.J.; REICHERT, J.M. \& KAISER, D.R. Compactação de um Latossolo induzida pelo tráfego de máquinas e sua relação com o crescimento e produtividade de feijão e trigo. R. Bras. Ci. Solo, 32:933942,2008 
DAVIES, W.J. \& ZHANG, J. Root signals and the regulation of growth and development of plant in drying soil. Ann. Rev. Plant Physiol. Plant Molec. Biol., 42:55-76, 1991.

DREW, M.C. Plant injury and adaptation to oxygen deficiency in the root environment: A review. Plant Soil, 75:179199, 1983.

EMPRESA BRASILEIRA DE PESQUISA AGROPECUÁRIA EMBRAPA. Centro Nacional de Pesquisa de Solos. Sistema brasileiro de classificação de solos. Rio de Janeiro: Embrapa Solos, 1999. 412p.

FONTANELA, E. Parâmetros físico-hídricos de um Latossolo sob diferentes sistemas de manejo e níveis de tráfego. Santa Maria, Universidade Federal de Santa Maria, 2008. 72p. (Tese de Mestrado)

GOODMAN, A.M. \& ENNOS, A.R. The effects of soil bulk density on the morphology and anchorage mechanics of the root systems of sunflower and maize. Ann. Bot., 83:293-302, 1999.

GreenWAY, H.; ARMSTRONG, W. \& COLMER, T.D. Conditions leading to high $\mathrm{CO}_{2}$ in waterlogged - flooded soils and possible effects on root growth and metabolism. Ann. Bot., 98:9-32, 2006.

IMHOFF, S.; SILVA, A.P.; DIAS JUNIOR, M.S. \& TORMENA, C.A. Quantificação de pressões críticas para o crescimento das plantas. R. Bras. Ci. Solo, 25:11-18, 2001.

KAISER, D.R.; REINERT, D.J.; ALBUQUERQUE, J.A.; RODRIGUES, M.F.; KUNZ, M. \& REICHERT, J.M. Condutividade de ar em Argissolo submetido a diferentes manejos e níveis de compactação. In: REUNIÃO BRASILEIRA DE MANEJO E CONSERVAÇÃO DO SOLO E DA ÁGUA, 17., Rio de Janeiro, 2008. Anais. Rio de Janeiro, SBCS, 2008. CD ROM.

KLEIN, V.A. \& LIBARDI, P.L. Densidade e distribuição do diâmetro dos poros de um Latossolo Vermelho sob diferentes sistemas de uso e manejo. R. Bras. Ci. Solo, 26:857-867, 2002.

KLEIN, V.A.; REICHERT, J.M. \& REINERT, D.J. Água disponível em um Latossolo Vermelho argiloso e murcha fisiológica de culturas. R. Bras. Eng. Agric. Amb., 10:646650, 2006.

KLEIN, V.A. \& CAMARA, R.K. Rendimento da soja e intervalo hídrico ótimo em Latossolo Vermelho sob plantio direto escarificado. R. Bras. Ci. Solo, 31:221-227, 2007.

KLUTE, A. Water retention: Laboratory methods. In: BLACK, C.A., ed. Methods of soil analysis. I. Physical and mineralogical methods. Madison, American Society of Agronomy, Soil Science Society of America, 1986. p.635662 .

LAPEN, D.R.; TOPP, G.C.; GREGORICH, E.G. \& CURNOEW, E. Least limiting water range indicators of soil quality and corn production, Eastern Ontario, Canada. Soil Till. Res., 78:151-170, 2004.

LEÃO, T.P.; SILVA, A.P.; MACEDO, M.C.M.; IMHOFF, S. \& EUCLIDES, V.P.B. Intervalo hídrico ótimo na avaliação de sistemas de pastejo contínuo e rotacionado. R. Bras. Ci. Solo, 28:415-423, 2004.
LETEY, J. Relationship between soil physical properties and crop productions. Adv. Soil Sci., 1: 277-294, 1985.

LOWERY, B. \& MORRISON, J.E. Soil penetrometers and penetrability. In: DANE, J.H. \& TOPP, G.C. Methods of soil analysis. Part 4: Physical methods. Madison, Soil Science Society of America, 2002. p.363-388.

MONTAGU, K.D.; CONROY, J.P \& ATWELL, B.J. The position of localized soil compaction determines root and subsequent shoot growth responses. J. Exper. Bot., 52:2127-2133, 2001.

MORARD, P. \& SILVESTRE, J. Plant injury due to oxygen deficiency in the root environment of soilless culture: A review. Plant Soil, 184:243-254, 1996.

RASSE, D.P. \& SMUCKER, A.J.M. Root recolonization of previous root channels in corn and alfalfa rotations. Plant Soil, 204:203-213, 1998.

REICHERT, J.M.; REINERT, D.J. \& BRAIDA, J.A. Qualidade dos solos e sustentabilidade de sistemas agrícolas. Ci. Amb., 27:29-48, 2003.

REICHERT, J.M.; SUZUKI, L.E.A.S. \& REINERT, D.J. Compactação do solo em sistemas agropecuários e florestais: Identificação, efeitos, limites críticos e mitigação In: CERRETA, C.A.; SILVA, L.S. \& REICHERT, J.M. Tópicos em ciência do solo. Viçosa, MG, Sociedade Brasileira de Ciência do Solo, 2007.v.5. p.49-134.

REICHERT, J.M.; SUZUKI, L.E.A.S.; REINERT, D.J.; HORN, R. \& HAKANSSON, I. Reference bulk density and crítical degree-of-compactness for no-till crop production in subtropical highly weathered soils. Soil Till. Res., 102:242254,2009 .

REINERT, D.J. \& REICHERT, J.M. Coluna de areia para medir a retenção de água no solo protótipos e testes. Ci. Rural, 36:1931-1935, 2006.

REINERT, D.J.; COLLARES, G.L \& REICHERT, J.M. Penetrômetro de cone com taxa constante de penetração no solo: desenvolvimento e teste de funcionalidade. Eng. Agríc., 27:304-316, 2007.

RIQUELME, U.F.B. Propriedades físicas e hídricas do solo em sistemas de manejo do feijoeiro. Santa Maria, Universidade Federal de Santa Maria, 2004. 78p. (Tese de Mestrado)

SCHNEIDER, P.R.; SCHNEIDER, P.S.P. \& SOUZA, C.A.M. Análise de regressão aplicada à engenharia florestal. Santa Maria, Universidade Federal de Santa Maria, 2009. $294 \mathrm{p}$.

SECCO, D.; REINERT, D.J. \& REICHERT, J.M. Produtividade de soja e propriedades físicas de um Latossolo submetido a sistemas de manejo e compactação. R. Bras. Ci. Solo, 28:797-804, 2004.

STIRZAKER, R.J.; PASSIOURA, J.B. \& WILMS, Y. Soil structure and plant growth: Impact of bulk density and biopores. Plant Soil, 185:151-162, 1996.

SILVA, V.R. Propriedades físicas e hídricas em solos sob diferentes estados de compactação. Santa Maria, Universidade Federal de Santa Maria, 2003. 171p. (Tese de Doutorado) 
SILVA, A.P. \& KAY, B.D. The sensitivity of shoot growth of corn to the least limiting water range of soils. Plant Soil, 184:323-329, 1996

SILVA, A.P. \& KAY, B.D. Estimating the least limiting water range of soils from properties and management. Soil Sci. Soc. Am. J., 61:877-883, 1997.

SILVA, A.P.; KAY, B.D. \& PERFECT, E. Characterization of the least limiting water range of soils. Soil Sci. Soc. Am. J., 58:1775-1781, 1994.

SILVA, V.R.; REINERT, D. \& REICHERT, J.M. Variabilidade espacial da resistência à penetração em plantio direto. Ci. Rural, 34:399-406, 2004.

STRECK, C.A. Compactação do solo e seus efeitos no desenvolvimento radicular e produtividade da cultura do feijoeiro e da soja. Santa Maria, Universidade Federal de Santa Maria, 2003. 83p. (Tese de Mestrado)
STRECK, C.A.; REINERT, D.J.; REICHERT, J.M. \& KAISER, D.R. Modificações em propriedades físicas com a compactação do solo causada pelo tráfego induzido de um trator em plantio direto. Ci. Rural, 34:755-760, 2004.

TAIZ, L. \& ZEIGER, E. Fisiologia vegetal. Porto Alegre, Artmed, 2004. 722p.

TORMENA, C.A.; SILVA, A.P. \& LIBARDI, P.L. Caracterização do intervalo hídrico ótimo de um Latossolo Roxo sob plantio direto. R. Bras. Ci. Solo, 22:573-581, 1998.

VEIGA, M.; HORN, R.; REINERT, D.J. \& REICHERT, J.M. Soil compressibility and penetrability of an Oxisol from Southern Brazil, as affected by long-term tillage systems. Soil Till. Res., 92:104-113, 2007.

WU, L.; FENG, G.; LETEY, J.; FERGUSON, L.; MITCHELL, J.; McCULLOUGH-SANDEN, B. \& MARKEGARD, G. Soil management effects on the nonlimiting water range. Geoderma, 114:401-414, 2003. 
